Sección uno: Ensayo

Radiografía de la innovación educativa en el Siglo XXI

\title{
Diseño de proyectos transmedia para la Educación Literaria en el aula de Educación Secundaria ${ }^{1}$
}

Design of transmedia projects for Literary Education in the Secondary Education classroom

\author{
José Hernández Ortega \\ Universidad Complutense de \\ Madrid \\ profesorhernandezortega@gmail \\ .com \\ José Rovira-Collado \\ Universidad de Alicante \\ jrovira.collado@gcloud.ua.es
}

\section{Resumen}

Entre las propuestas de innovación educativa del siglo XXI, el Aprendizaje Basado en Proyectos (ABP) se ha convertido en una metodología colaborativa global en la que pueden integrarse distintas asignaturas y aprendizajes, con gran repercusión en la etapa de Educación Secundaria. Aunque los proyectos no deben estar mediados por la tecnología, los que la incluyen como eje para la producción de contenidos y la difusión de estos se convierten en proyectos con mayor reconocimiento y con muchas posibilidades de replicación. El concepto de Educación 2.0 es imprescindible para reconocer la importancia de compartir nuestro trabajo en Internet a través de blogs y redes. La incorporación del concepto de Narrativas Transmedia a estos proyectos nos permite enlazar directamente esta deriva tecnológica con la Educación Literaria. Los proyectos transmedia parten de la lectura de obras literarias para ofrecer múltiples producciones por parte del alumnado que integran los distintos aprendizajes.

Palabras Clave: Aprendizaje Basado en Proyectos (ABP), Narrativas Transmedia, Lectura Multimodal, Didáctica de la Literatura, Educación 2.0.

${ }^{1}$ Recibido: 15/01/2020 Evaluado: 29/01/2020 Aceptado: 24/02/2020 


\begin{abstract}
Among the proposals for educational innovation of the 21st century, Project Based Learning (PBL) has become a global collaborative methodology in which different subjects and learning can be integrated, with great impact on the Secondary Education. Although the projects should not be mediated by technology, those that include it as an axis for the production of content and the dissemination of these become projects with greater recognition and with many possibilities of replication. The concept of Education 2.0 is essential to recognize the importance of sharing our work on the Internet through blogs and networks. The incorporation of the concept of Transmedia Narratives to these projects allows us to directly link this technological drift with Literary Education. The transmedia projects start from the reading of literary texts to offer multiple productions by the students that integrate the different learning.
\end{abstract}

Keywords: Project Based Learning (PBL), Transmedia Narratives, Multimodal Reading, Literature Teaching, Education 2.0.

\title{
Introducción
}

La vigente tendencia en innovación educativa conduce a una revisión de los planteamientos realizados hasta la fecha. Innovar se concibe como una transformación de los espacios educativos digitales que se hallan en constante transformación y evolución. El proceso de digitalización de los contextos escolares requiere de una planificación y puesta en práctica que no siempre se realiza con la distancia y el proceso adecuado. Aquellos centros que anteponen la incorporación de la tecnología al diseño de un plan de desarrollo innovador están abocados a un fracaso, cada vez más corroborado por prácticas pseudo-innovadoras poco efectivas desde la óptica pedagógica (García-Peñalvo, 2015; Area, Hernández y Sosa, 2016, 2019; Meneses, Fàbregues, Jacovkis y Rodríguez-Gómez, 2014; Kampylis, Devine, Punie y Newman, 2016).

Siguiendo las investigaciones de Area, Cepeda y Feliciano (2018) existe una correlación directa entre la visión de las TIC que tienen independientemente docentes y alumnado y el proceso de innovación. Innovar, como idea vinculada a los conceptos mejora, cambio y avance (Ferrer, 2017; Iglesias, Lozano y Roldán, 2018), necesita de una adecuada planificación pedagógica, donde la formación del profesorado es el eje sobre el que construir políticas innovadoras que repercutan en las aulas de forma coherente. Tecnologías emergentes, metodologías activas, aprendizaje cooperativo, pensamiento crítico, Aprendizaje Basado en Proyectos $(A B P)$, Gamificación, Aprendizaje Basado en el Pensamiento o Aprendizaje-Servicio son los estandartes contemporáneos del proceso de innovación educativa en todas las áreas de conocimiento. 
En esta investigación se hace un recorrido por los conceptos de lectura multimodal y narrativas transmedia para mostrar cómo han transformado la Didáctica de la Lengua y la Literatura (DLL) en los últimos años, sobre todo con la sobrexposición constante a Internet. A partir de aquí se señala la necesidad de utilizar las herramientas 2.0 como blogs y redes sociales para la divulgación de la innovación educativa y como todas estas herramientas son indispensables para el diseño de proyectos transmedia para el aula de Educación Secundaria. Los proyectos de trabajo son una propuesta innovadora para la Educación Literaria (Margallo, 2012) porque nos permiten recoger las producciones del alumnado a partir de las distintas lecturas que se realicen. Además, la cantidad de información generada en estos proyectos y su publicación en la red nos permiten realizar una completa investigación en las aulas, una de las tendencias imprescindibles para la DLL en el siglo XXI (Sánchez y Cancelas, 2015).

\section{El valor del componente transmedia en la innovación en la DLL}

En el contexto de una sociedad multimodal (Kress, Jewitt, Ogborn, y Tsatsarelis, 2014), las estrategias que apuestan por la innovación en la didáctica específica de la DLL lo hacen, de forma ineludible y necesaria por los elementos multimodales o transmedia (Scolari, 2013). La comunicación verbal ha pasado de ser monodimensional a un amplio ecosistema de formatos que aumentan el valor semiótico del lenguaje y del acto comunicativo.

Las múltiples posibilidades que ofrece la multimodalidad amplían cualitativamente el valor de la narración literaria y dotan de mayor carga semiótica a los procesos comunicativos, informativos y lecto-escritores (Kress y Van Leeuwen, 2001; Van Leeuwen y Jewitt, 2001). El incremento semiótico del mensaje literario, especialmente en su aplicación al contexto educativo, adquiere su máxima expresión a partir de la presencia de Internet. El uso de redes sociales $\mathrm{y}$, especialmente, de dispositivos móviles genera nuevos ecosistemas para la creación de productos comunicativos y de medios de interacción entre la obra literaria y los lectores, convertidos estos en prosumidores de metaliteratura. García-Ruiz, Ramírez García y Rodríguez Rosell acotan su definición: “un ciudadano prosumidor será poseedor de una serie de competencias que le permitirán llevar a cabo un conjunto de acciones, tanto como consumidor de medios y recursos audiovisuales, como productor y creador de mensajes y contenidos críticos, responsables y creativos” (2014, p. 16). Este nuevo paradigma didáctico obliga a una reflexión del proceso de aprendizaje en un nuevo ecosistema del aprendizaje de la Lengua y Literatura:

Los enfoques multimodales, es decir, aquellos que tienen en cuenta esta diversidad de modos de comunicar, reconocen que el proceso de enseñanza-aprendizaje es una actividad que ya no está únicamente centrada en el lenguaje, sino que los gestos, las imágenes, el sonido, las acciones constituyen elementos de representación muy valiosos que participan en este proceso. La mirada multimodal sobre la comunicación favorece el nuevo paradigma educativo que destaca la estrecha vinculación que existe entre el aprender y el hacer y la necesidad de diseñar contextos de aprendizaje apropiados que posibiliten el desarrollo de prácticas educativas auténticas, coherentes y significativas, es decir, que sean culturalmente relevantes y constituyan una actividad social. (Cuestas y Valotta, 2011, p. 3) 
Aunque la concepción del término es anterior, la definición de Narrativas Transmedia (NT) por Henry Jenkins en 2008 y el posterior desarrollo de Carlos Scolari (2013) nos permiten identificar las nuevas formas de consumo audiovisual del siglo XXI. También el concepto de lectoespectador (Mora, 2012) nos indica la necesidad de relacionar la lectura literaria con las propuestas audiovisuales del siglo XXI, encauzadas la mayoría a través de la pantalla de un ordenador o de un móvil.

Obras de referencia en los últimos años son, entre una amplia selección, las sagas de Star Wars, Harry Potter, el Señor de los anillos, Juego de Tronos... y un amplio etcétera que han dado el salto a la gran pantalla, televisión, videojuegos, campañas de mercadotecnia, han mutado en spin-offs y/o fan-fictions... a partir de relatos literarios, o en sentido inverso, han generado novelas de franquicia y una gran influencia en la cultura popular a través de las propuestas cinematográficas.

En la literatura juvenil española contemporánea asistimos a la producción, cada vez más frecuente, de títulos que desarrollan itinerarios multimodales paralelos a la trama argumental de la ficción literaria (listas de Spotify, canales de Youtube, perfiles en redes sociales, etc.). Desde Todos los detectives se llaman Flanagan, (Ribera y Martín, 2005) a Canciones para Paula (Jeans, 2011), pasando por Xènia, tens un WhatsApp (Pasqual, 2014), Canciones de amor a quemarropa (Butler, 2014), Todos mis futuros son contigo (Abu-Tahoun, 2015) o Pulsaciones (Ruescas, 2014) la transición del papel a la red se produce cada vez de forma más prematura en la propia trama argumental y, por ende, más allá de las páginas del propio libro. Estas dinámicas responden a los intereses del alumnado y sus prácticas digitales habituales. Una propuesta innovadora puede no ser digital, pero no puede ir en contra de los intereses actuales de los jóvenes.

Una propuesta concreta de narrativa transmedia en la Literatura Infantil y Juvenil (LIJ) española fue la saga Odio al Rosa https://bit.ly/2WdCenx. Escrita por Ana Alonso y Javier Pelegrín e ilustrada por Miguel Navia y Esther Lecina, este proyecto conjuga el relato adolescente con las múltiples posibilidades de interacción que nos ofrece Internet. En los últimos años, una experiencia transmedia para el gran público que conjuga literatura e historia ha sido la serie de Televisión Española El Ministerio del Tiempo, de Pablo y Javier Olivares. El diseño de producción de la serie está pensado como un gran entramado transmedia, con expansiones en Internet, novelas, juegos y cómics, y ha dado espacio a muchas propuestas educativas (Gómez-Trigueros, Rovira-Collado y Ruiz-Bañuls 2018; Scolari, Lugo y Masanet, 2019; Zhang y Cassany 2019).

Esta multiplicidad de formatos favorece que la apuesta por la interpretación de esta tipología de obras en particular, pero de cualquier obra literaria en general, sea susceptible de una reinterpretación basada en estrategias multimodales que no solo atesoren la comprensión lectora, sino una producción supracomunicativa por parte de los lectores.

Aunque el concepto de NT se circunscribe a la expansión de un relato por múltiples soportes o plataformas, hasta la configuración de universo narrativo propio, creemos conveniente aplicar este sentido a los proyectos que presentaremos, ya que la lectura se traslada a otros soportes. Para el diseño de ABP es necesaria la formación del profesorado en las narrativas 
transmedia para poder aplicar su competencia digital en futuros proyectos (GómezTrigueros, Ruiz-Bañuls y Ortega-Sánchez, 2019).

\section{Didáctica de la Literatura 2.0 o la necesidad del profesorado conectado}

Desde su aparición, el término 2.0 se ha utilizado muchas veces como sinónimo de innovación, añadiendo el significado de novedad o transformación al sustantivo al que se añadía. Cocina 2.0, Política 2.0 o Educación 2.0 son términos que hemos escuchado en estas dos décadas del siglo XXI como ejemplos de nuevas formas de hacer las cosas o ruptura con los modelos anteriores, aunque muchas veces se limitaban a introducir un uso superficial de las TIC. Debemos anotar que este término se refiere a las nuevas posibilidades de la web social y es importante conocer lo que supone su transformación para la generación del conocimiento. Ahora cualquier persona puede crear su espacio en Internet y debe ser consciente de su identidad digital (Castañeda y Camacho, 2012). Aunque el término 2.0 puede haber sido ya superado, por el concepto 3.0 respecto a la web semántica o el 4.0 para referirse a la web ubicua, los planteamientos de la web social han sido fundamentales en la transformación de la didáctica en los últimos veinte años. No significa que tengamos un blog o que participemos en muchas redes sociales. La Didáctica de la Literatura 2.0 nos permite crear nuevos contenidos, compartirlos en abierto en la red con otras muchas personas, comentarlos y reutilizarlos para nuestras clases, configurando de este modo un entorno personal de aprendizaje (PLE) especializado en el ámbito de la DLL en la era digital (RoviraCollado y Hernández Ortega, 2016). No es la tecnología, sino las dinámicas 2.0 lo que han supuesto una transformación en la educación.

En anteriores investigaciones hemos anotado las posibilidades de los blogs para el desarrollo de la competencia docente de nuestro alumnado (Rovira-Collado, 2016), recogiendo un listado de blogs como ejemplos de buenas prácticas para la DLL y la LIJ. Entre otros, podemos señalar la importancia de los espacios de Fernando Trujillo https://bit.ly/3bIspV8, Toni Solano https://bit.ly/2xaXvWn, o del desaparecido Felipe Zayas https://bit.ly/3eU6Ene, como claros ejemplos de la transformación que los blogs han supuesto para la DLL.

También podemos destacar las enormes posibilidades educativas de las redes sociales. Ya en 2011 tenemos una amplia descripción de las posibilidades de Twitter para el desarrollo de la competencia comunicativa y la competencia digital (Lara, 2011):

Tanto Twitter como otras herramientas digitales propias de la web 2.0 son espacios de interacción que deben ser comprendidos y utilizados como un complemento ideal a la canalización del trabajo en grupo y como vías para dinamizar los debates con una mayor necesidad de profundización en otros espacios más adecuados para ello (blogs, foros, etc.). Para poder aprovechar todo su potencial es imprescindible que los profesores conozcan estos espacios y que los practiquen como usuarios activos, de modo que puedan guiar a sus alumnos en el uso estratégico más significativo para el desarrollo de sus materias. (Lara, 2011, p. 46). 
La interacción entre ambas herramientas es indispensable. Más allá del uso de otras estrategias didácticas como Youtube (Pérez-Torres, Pastor Ruiz y Abarrou-Ben-Boubaker, 2018; Aran-Ramspott, Fedele y Tarragó, 2018; Lluch, 2019), el blog (Heredia y Romero, 2017) y las redes sociales (Corpas y Rubio, 2017) son dos herramientas imprescindibles para cualquier docente que se considere innovador. Es cierto que ya han aparecido nuevas dinámicas en Internet relacionadas con la lectura como los booktuber y otros epitextos digitales (Álvarez y Romero, 2018), que aprovechan la vertiente audiovisual de la red y sus altas dosis de influencia en el alumnado adolescente, pero las redes siguen siendo la vía de comunicación y los blogs, el espacio para recoger todas las propuestas y presentarlas de una forma organizada.

Para acceder a la infinidad de nuevas voces en la DLL 2.0 es necesario seguir en redes al profesorado que recoge en los blogs, de una forma estructurada, sus propuestas para el aula de lengua y literatura. En la siguiente lista de Twitter https://bit.ly/3cLGh0T encontramos más de 300 perfiles de docentes de lengua y literatura. Para conocer algunos ejemplos de nuevos espacios, podemos visitar los siguientes blogs, que también incluyen la metodología basada en proyectos:

- https://bit.ly/2S8SEMB de Alberto Corpas Martos,

- https://bit.ly/3aFCwIE de Raúl Rubio Millares,

- https://bit.ly/2KD5PRE de Antonio Garrido.

En una reciente experiencia didáctica compartida con profesorado ecuatoriano (RoviraCollado, Ambròs y Hernández Ortega, 2019), nuestro objetivo fue mostrar las enormes posibilidades de ambas herramientas para posteriormente poder embarcarnos en el desarrollo de proyectos transmedia.

\section{El Aprendizaje Basado en Proyectos: herramienta para la innovación en DLL}

La elección del Aprendizaje Basado en Proyectos (ABP) como eje de nuestro análisis, además de acertada y necesaria, quiere situar a las metodologías activas en el centro del proceso de innovación y mejora educativa para una adquisición óptima de las competencias (De la Cruz, 2003; Fernández, 2006), en especial las referidas a la potenciación de la "creatividad, resolución de problemas, habilidad de investigar, trabajar de forma colaborativa, motivación y uso de redes sociales [...]” (Ausín, Abella, Delgado, y Hortigüela, 2016, p. 32) como también del "pensamiento crítico, la capacidad de autorregulación del propio proceso de aprendizaje [...], la solución de problemas o la integración comprensiva del pensamiento” (Badia y García, 2006, p. 48) pero es, fundamentalmente, un proceso a través del cual se aprende a aprender, adquiriendo "un valor especial de relevancia en la medida que debemos promover en el alumnado procesos educativos en los que aprendan a construir y gestionar el conocimiento” (Cascales, Carrillo, y Redondo, 2017, p. 203). Enfoque a partir del cual podemos hablar de una variedad en las distintas estrategias didácticas de estas metodologías, como así han enumerado Rekalde y García (2015, p. 221) en una acertada disección del espectro metodológico activo liderado por el Aprendizaje Cooperativo, el Aprendizaje Basado en la Investigación, el Aprendizaje y Servicio Solidario, el Aprendizaje Basado en Problemas y el Aprendizaje Basado en Proyectos. 
El eje principal de esta corriente es, de forma axiomática, el alumnado, sobre quien gira un proceso complejo que busca, procesa, edita, mejora y consolida su propio conocimiento a través de la práctica activa y directa sobre el proceso. A fin de convertir este marco teórico en una sustancia tangencial, el alumno debe transformar los aspectos teóricos en práctica activa a través de contextos reales que le alimenten tanto de conocimiento como de la propia perspectiva del rol que ejerce. En este marco, la multimodalidad proporciona un conjunto de posibilidades que facilitan que el alumnado exponga sus capacidades, inquietudes e interpretaciones de la obra literaria a partir de las propias capacidades, así como de otros conocimientos instrumentales adquiridos, principalmente, a través de la red.

La apuesta didáctica por esta estrategia pedagógica en la DLL está en un constante crecimiento, mejora y consolidación. Prueba de ello se halla en la recopilación de proyectos de base transmedia, basados o no en la presencia tecnológica, realizada en el Portfolio lector https://bit.ly/3bDBPkw donde se pueden hallar más de un centenar de propuestas de ABP en la DLL, tanto en el marco escolar de la LIJ como de la literatura en su dimensión general. De entre ellos, podemos destacar ABP de referencia que se desarrollan en ámbitos interdisciplinares y que ofrecen valores significativos en la dimensión transmedia.

\section{Rutas literarias como geolocalización lectora}

Para comenzar nuestro análisis, las rutas literarias se erigen como una de las apuestas más consolidadas en la implementación de la educación multimodal a través del ABP. El espacio de creación trasciende las barreras físicas del aula para la adquisición del conocimiento en espacios físicos próximos a la vida real del alumnado. Recuperar las principales obras de nuestra literatura a través de las calles de nuestros pueblos y ciudades es el objetivo de estos proyectos colaborativos, donde la participación del alumnado es imprescindible (Cadenato y Domenech, 2019). La literatura sale de las aulas y se adueña de la calle, dando un sentido real a la Educación Literaria al relacionarla con los espacios más cercanos. Alexandre Bataller (2014) hace un completo recorrido por las propuestas que usan la tecnología para localizar la literatura para conocer en qué medida la geolocalización de obras literarias y el aprendizaje colaborativo del alumnado a través de plataformas digitales favorece el desarrollo de las distintas competencias y de la Educación Literaria. Destacamos una selección de proyectos que constituyen apuestas metodológicas de referencia por su valor multimodal y de construcción del conocimiento interdisciplinar a partir de los conocimientos propios del área de DLL:

Callejeros literarios: http://bit.ly/2NrrcYf. Lanzado en el curso 2010-2011 por los autores y autoras de cuatro de los blogs educativos más relevantes A pie de Aula, Blogge@ndo, $\underline{\operatorname{Re}(p a s o)}$ de Lengua y Tres Tizas es un proyecto pionero (Fernández Campos, González Mendizábal y Pérez Gómez, 2012) y, posiblemente, el modelo más completo en este ámbito. Es un proyecto colaborativo donde se comparten las experiencias de múltiples centros, que crecen año a año. En la primera edición participaron 29 centros de 12 comunidades autónomas (País Vasco, Cataluña, Comunidad de Madrid, Comunidad Valenciana, Murcia, Navarra, Andalucía, Castilla-León, La Rioja, Castilla-La Mancha, Galicia, Islas Baleares) y se realizaron 34 callejeros de diferentes ciudades. Fue un proyecto plurilingüe con propuestas redactadas en castellano, euskera y catalán. Participaron casi novecientos alumnos de Primaria, Secundaria y Bachillerato. Este proyecto demuestra la importancia del aprendizaje

Nº 8, 2020. Página | 86 
colaborativo y cómo se pueden imitar las enseñanzas de éxito de una forma global y ofreciendo la información en abierto en Internet.

REDsidencia de Estudiantes: http://bit.ly/3069vCt. Es un proyecto colaborativo de acercamiento a la poesía de la Generación del 27 realizado durante el curso 2011-2012 por el $2^{\circ}$ de Bachillerato A del IES V Centenario de Sevilla. El proyecto "trata de imaginar cómo serían, en la actualidad, las relaciones entre los amigos que formaron este grupo. Posiblemente aprovecharían las nuevas tecnologías para compartir ideas, textos, fotografías, vídeos... Posiblemente participarían en alguna red social para estar en contacto en todo momento, utilizando para ello los distintos dispositivos móviles a nuestro alcance”. Fue Premio Fundación Telefónica de Innovación Educativa en 2012 y es una suerte que todavía esté disponible en la red, para poder acercar la poesía del 27 a los jóvenes lectores.

El Instagram de Luis Cernuda: http://bit.ly/35FPjsh. Más reciente, este proyecto para la asignatura Lengua Castellana y Literatura de $4^{\circ}$ de ESO y $2^{\circ}$ de Bachillerato del IES Chaves Nogales también de Sevilla, se centra en la obra del autor de Ocnos y La Realidad y el Deseo y la relación con su ciudad. Coordinado por los profesores Diego Bernal y Nacho Gallardo, este proyecto demuestra que la evolución de la tecnología es imparable y donde antes se usaba Twitter, ahora el alumnado solo conoce Instagram. La relación entre fotografías y textos literarios es otra clave de las rutas literarias.

Rutas Geonarrativas: http://bit.ly/2T4EPjA. También más actual, este proyecto incorpora nuevas herramientas como Google Tour Builder. Es un proyecto para la Academia de Innovadores de Google 2018 de Domingo Chica Pardo, profesor en el Colegio San José en Vélez-Málaga. Encontramos actividades con el alumnado de inglés de primer curso de la ESO. En la presentación plantea las siguientes preguntas, que relacionan el ABP y el desarrollo de las competencias clave con la lectura literaria y podrían aplicarse a cualquiera de los proyectos analizados.

¿Cómo podríamos conseguir que el alumnado trabaje las competencias clave a lo largo de la lectura?

¿Cómo podríamos diseñar proyectos para que un libro de lectura se convierta en toda una experiencia de aprendizaje?

¿Cómo podríamos integrar la tecnología en nuestra propuesta con el fin de secuenciar el proceso y ubicar espacios narrativos para hacerlos más visuales?

World Mobile City Project https://bit.ly/3eQpk7f es otro proyecto colaborativo entre distintos centros escolares, lanzado en 2014 para hacer propuestas geolocalizadas en varias ciudades. Surge a partir del proyecto de Barcelonada-WMCProject: http://bit.ly/2QBOYT4 organizado por la red de docentes Lacenet https://bit.ly/3aGIFVb. La red ha ido creciendo a lo largo de los años y encontramos información detallada de múltiples actividades, como por ejemplo la Gaiztekada-WMCProject: http://bit.ly/36Dc3dG o la Alcoianada-WMCProject: http://bit.ly/2uzTvgq, dedicada en 2017 al poeta Joan Valls. El profesor Juan Francisco Álvarez ha coordinado varios encuentros y destaca la importancia para el desarrollo de la 
competencia digital docente y del alumnado (Larraz Rada, Álvarez Herrero, Espuny Vidal, González Martínez, 2019). Es un proyecto riquísimo que demuestra la necesidad de utilizar elementos reales en la educación y justifica el uso del móvil para muchas actividades, frente a otras propuestas que quieren restringir su uso, cerrando esta ventana al mundo.

\section{ABP con diseño transmedia y multimodal}

La integración del currículum académico correspondiente a Lengua y Literatura en Educación Secundaria con una propuesta de intervención didáctica multimodal posibilita una heterogénea y amplia variedad de actividades, como hemos visto previamente, donde caben todas las competencias clave. En un plano más concreto, podemos destacar los siguientes proyectos desarrollados a lo largo de los años, coordinados por José Hernández Ortega, uno de los autores de esta investigación y realizados en distintos cursos de Colegio El Valle de Madrid. En ellos se persigue que la innovación no se consiga a través de una herramienta digital pasajera, sino que el valor sustancial innovador se plantee a partir de mecanismos y planteamientos que, de otra forma, no hubiesen proporcionado un aprendizaje significativo y activo para el alumnado, donde la creatividad y la motivación son elementos transversales a todo el proceso.

ABP Cielo abajo: http://bit.ly/39Vf7DY. Realizado junto con la profesora Beatriz Martín con el alumnado de $2^{\circ}$ de la ESO del curso 2013-2014, se basa en los trabajos que realizó el alumnado sobre la obra Cielo Abajo de Fernando Marías (2005), que recibió el Premio Nacional de Literatura Infantil y Juvenil en 2006. En los agentes implicados para este proyecto destacamos la lectura literaria, el uso de la tecnología educativa, la investigación y el trabajo compartido por los distintos grupos y la importancia de la oralidad para conocer la obra. También destacan las dinámicas de evaluación y coevaluación que se proponen.

ABP Emocionario literario: https://bit.ly/2VX33fv. La siguiente propuesta está coordinada por Pep Hernández, Beatriz Martín del área de Lengua y por Ana Alfonsel (Música) y Ana Gil (Plástica), demostrando la importancia de la interdisciplinariedad en estos proyectos y fue realizada con los cursos de $1^{\circ}$ y $2^{\circ}$ de la ESO del curso 2016-2017. En este proyecto se trabaja la transversalidad literaria y lingüística en dos proyectos correspondientes a los dos cursos que lo conforman. A lo largo del curso se trabajó la temática emocional derivada de la interpretación literaria de dos de las lecturas obligatorias programadas. Encontramos una completa guía didáctica que justifica la propuesta y nos permitiría repetir la experiencia en otros centros. La cantidad de trabajo realizado por el alumnado sobre las obras trabajadas y disponible en la web nos confirman que esta metodología favorece la comprensión y el interés por los textos literarios trabajados, aunque sean obligatorios. Una de las obras analizadas en este proyecto nos lleva al siguiente.

ABP El libro de la selva: stop motion edition: http://bit.ly/35DTNzF. Este proyecto ofrece la conexión entre literatura, lenguaje audiovisual (cinematográfico) y la interacción con áreas curriculares científicas como Matemáticas o Tecnología para el desarrollo global de competencias comunicativas y digitales del alumnado. El equipo docente crece y las posibilidades se enriquecen con la aportación de distintas asignaturas. Se pueden comparar las propuestas dedicadas a esta obra en el curso anterior con estos nuevos vídeos, que adaptan los distintos episodios de la novela. La participación de docentes del área de Lengua Inglesa 
permite acercarnos al texto desde un enfoque plurilingüe. La rápida evolución de la tecnología también se observa en el diseño de la página y en la edición de los materiales multimodales.

ABP Soy leyenda: http://bit.ly/36GaGLt. En este proyecto de 2019 el alumnado se convierte en Reales Cronistas de la Villa y Corte de Madrid. A través de anécdotas, leyendas e historias nos ofrecen creaciones transmedia para disfrutar de la ciudad, y trabajar contenidos lingüísticos, literarios e históricos del Madrid más próximo para los adolescentes. Además de Lengua Castellana y Lengua Inglesa participan los docentes de Geografía e Historia, con un equipo de ocho docentes, ofreciendo una guía turística completa y adecuada para todos los públicos, pero sobre todo para el juvenil, porque los contenidos están creados por alumnado de la ESO.

ABP La horda del lector: http://bit.ly/35FNiwi. Al anterior proyecto se suma este, donde se aglutinan las distintas propuestas transmedia a través de diversos formatos audiovisuales para el fomento y la animación a la lectura en ESO. La cantidad del trabajo realizado por el alumnado es abrumador y lo podemos encontrar en las distintas secciones: Fotoportadas; Booktubers; Booktráilers; Lectores ilustres, trabajando distintas dinámicas de adaptación de textos literarios a la realidad digital. La presencia en redes sociales es constante, como la dinámica de BookFace en Instagram, recogida en https://bit.ly/2zuSx7v

Cada uno de estos proyectos alberga la mayor parte de los trabajos realizados por los distintos grupos a lo largo de los años. La cantidad de propuestas recogidas excede las posibilidades de análisis de este trabajo, pero se ha realizado un seguimiento a lo largo de los años que confirma la mejora progresiva de los resultados de los distintos grupos. Estos proyectos se configuran como un portafolio docente que también recoge las competencias desarrolladas por el alumnado.

\section{Conclusiones}

La actual corriente de innovación educativa requiere de la reflexión sobre los elementos constitutivos en el actual marco pedagógico, donde convergen múltiples factores que condicionan la apuesta por cualquiera de los itinerarios a disposición del profesorado de cualquier etapa educativa. La DLL se halla en un claro proceso de renovación pedagógica en el que encuentra en los nuevos planteamientos didácticos su fuente de crecimiento y expansión instrumental y procedimental. Las metodologías activas como el ABP o el uso del Transmedia son necesarias en la formación del profesorado actual (Gómez-Trigueros, RuizBañuls, 2017), pero son todavía más importantes en la etapa de Educación Secundaria, ya que el alumnado demuestra un gran interés en estas y ya tiene las destrezas necesarias para participar en el desarrollo de los proyectos.

En este contexto, la apuesta por la multimodalidad discursiva tanto docente como discente, cobra una importante relevancia a través de las posibilidades que ofrecen desde la creación de blogs educativos, el desarrollo de las redes sociales con fines educativos y su culminación más reciente en Aprendizaje Basado en Proyectos (en cualquiera de sus formas). Mecanismos de creación semiótica que ofrecen una expansión multidimensional que cobra nuevos significantes en paralelo al desarrollo de la semiótica amparada en la tecnología evolutiva 
(Scolari, 2013; Kress, Jewitt, Ogborn, y Tsatsarelis, 2014). Asimismo, junto al desarrollo tecnopedagógico se gestiona la transformación del paradigma educativo, centrado en el fomento de las competencias del alumnado para la consecución de un aprendizaje significativo y duradero (Cascales, Carrillo y Redondo, 2017). La participación del alumnado en esta Didáctica 2.0 es imprescindible y en la etapa de secundaria es necesario unir la competencia en comunicación lingüística con la digital para mejorar los aprendizajes. Aprendizajes que tienen en la tecnología la piedra axial para la concepción de la información, la innovación y la evolución pedagógica en un contexto social altamente digitalizado que todavía debe tener una correlación en el ámbito educativo (Area, Cepeda y Feliciano, 2018; Kampylis, Devine, Punie y Newman, 2016; De la Cruz, 2003).

En el ámbito universitario podemos encontrar infinidad de proyectos de innovación educativa en todos los cursos y áreas de conocimiento. Pero es en la etapa de Educación Secundaria donde encontramos los proyectos más elaborados respecto a la Didáctica de la Literatura 2.0.

\section{Agradecimiento}

Esta investigación está dentro de la Red de Investigación de Docencia Universitaria del ICE de la Universidad de Alicante: Alfabetización transmedia y lectura multimodal en el estudio de la Literatura Infantil y Juvenil. Innovación digital y LIJ 2.0 en asignaturas de Didáctica de la Lengua y la Literatura (4644).

\section{Referencias}

Abu-Tahoun, M. (2015). Todos mis futuros son contigo. Barcelona, Planeta.

Álvarez Ramos, E. y Romero Oliva, M. F. (2018). Epitextos milénicos en la promoción lectora: morfologías multimedia dela era digital. Revista Letral, n. ${ }^{\circ}$ 20, 71-85. DOI: http://dx.doi.org/10.30827/RL.v1i20.7830.

Aran-Ramspott, S., Fedele, M. \& Tarragó, A. (2018). YouTubers' social functions and their influence on pre-adolescence. [Funciones sociales de los Youtubers y su influencia en la preadolescencia]. Comunicar, 57, 71-80. DOI: https://doi.org/10.3916/C572018-07.

Area Moreira, M., Cepeda Romero, O., \& Feliciano García, L. (2018). El uso escolar de las TIC desde la visión del alumnado de Educación Primaria, ESO y Bachillerato. Educatio Siglo XXI, 36, 229-254. DOI: https://doi.org/10.6018/j/333071.

Area, M, Hernández, V. M. \& Sosa, J.J. (2019). Leadership and school integration of ICT. Teachers perceptions in Spain. Educ Inf Techno, 24, 549-565. DOI: https://doi.org/10.1007/s10639-018-9789-0. 
Area, M., Hernández, V. \& Sosa, J.J. (2016). Models of educational integration of ICTs in the classroom. [Modelos de integración didáctica de las TIC en el aula]. Comunicar, 47, 79-87. DOI: https://doi.org/10.3916/C47-2016-08.

Ausín, V., Abella, V., Delgado, V. y Hortigüela, D. (2016). Aprendizaje Basado en Proyectos a través de las TIC. Una experiencia de Innovación Docente desde las Aulas Universitarias. Formación Universitaria, 9(3), 31-38. Recuperado de: $\quad$ https://cutt.ly/YozvYcd,DOI: http://dx.doi.org/10.4067/S071850062016000300005.

Badia, A. y García, C. (2006). Incorporación de las TIC en la enseñanza y el aprendizaje basados en la elaboración colaborativa de proyectos. En: Antoni Badia (coord.) Enseñanza y aprendizaje con TIC en la educación superior [monográfico en línea]. 450 Revista de Universidad y Sociedad del Conocimiento (RUSC). 3 (2). https://cutt.ly/1oxXiKH.

Bataller, A. (2014). Hipertextos literaris georeferenciats i dispositus didàctics per a l'estímul de la Competència literària. En O.Cleger, J.M De Amo. La educación literaria y la e-literatura desde la minificción. Enfoques hipertextuales para el aula. Barcelona: Universitat de Barcelona Institut de Ciències de l’Educació. pp. 334-346.

Butler, N. (2014). Canciones de amor a quemarropa. Barcelona: Libros del asteroide.

Cadenato Matía, M. y Domenech Cases, L. (2019). Callejeros literarios. Peonza: Revista de literatura infantil y juvenil, No 129 (Lecturas de la ciudad), 37-43.

Cascales, A., Carrillo, M. E. y Redondo, A. M. (2017). ABP y Tecnología en Educación Infantil. Pixel-Bit. Revista de Medios y Educación, 50, 201-210. DOI: http://doi.org/c6q5.

Castañeda, L. y Camacho, M. (2012) Desvelando nuestra identidad digital”. El profesional de la información, julio-agosto, v. 21, n. 4, 354-360.

DOI:http://dx.doi.org/10.3145/epi.2012.jul.04.

Corpas Martos, A., y Rubio Millares, R. (2017). Expandiendo el aula a través del microblogging. Revista de Estudios Socioeducativos. ReSed, (5), pp. 119-129. Recuperado de https://cutt.ly/1ozcPIp.

Cuestas, A. D. y Valotta, M. E. (2011). Alumnos y docentes 2.0: Interacción y multimodalidad en la enseñanza de lenguas extranjeras, Revista Puertas Abiertas, 7. Recuperado de: https://cutt.ly/KozxNws

De la Cruz, M. A. (2003). Taller sobre el proceso de aprendizaje-enseñanza de competencias. Zaragoza: Instituto de Ciencias de la Educación, Universidad de Zaragoza. 
Fernández Campos A., González Mendizábal, I. y Pérez Gómez, MM. (2012) Callejeros literarios: una propuesta para la educación literaria. Revista Iberoamericana de Educación. Monográfico Didáctica de la Lengua y la Literatura, n.59. pp. 157-168.

Fernández March, A. (2006). Metodologías activas para la formación de competencias. Educatio siglo XXI, 24, pp. 35-56. Recuperado de: https://cutt.ly/Wozb1Oy

Ferrer, A. (2017). VirPLC: una metodología para el desarrollo de capacidades, habilidades y autoestima mediante la estimulación de la lógica con una herramienta sencilla, funcional y dinámica. Education In The Knowledge Society (EKS), 18(2), 59-69. DOI: https://doi.org/10.14201/eks20171825969.

García-Peñalvo, F. J. (2015). Mapa de tendencias en Innovación Educativa. Education in the Knowledge Society. 16 (4), 6-23. Recuperado de: https://cutt.ly/sozbgGo

García-Ruiz, R., Ramírez García, A. Rodríguez Rosell, M. M. (2014). Educación en alfabetización mediática para una nueva ciudadanía prosumidora. Comunicar,43 (vol. 24), 15-23 DOI: https://doi.org/10.3916/C43-2014-01.

Gómez-Trigueros I. M. y Ruiz-Bañuls M. (2017). Análisis de metodologías activas con ABP, Transmedia y Gamificación para implementar las competencias en Ciencias Sociales y en Literatura. En R. Roig-Vila, (ed.). Investigación en docencia universitaria. Diseñando el futuro a partir de la innovación educativa. Barcelona: Octaedro, pp. 245-254.

Gómez-Trigueros, I. M., Rovira-Collado, J. y Ruiz-Bañuls, M. (2018). Literatura e Historia a través de un universo transmedia: posibilidades didácticas del Ministerio del Tiempo. Revista Mediterránea de Comunicación/Mediterranean Journal of Communication. 9 (1), 217-225.

https://www.doi.org/10.14198/MEDCOM2018.9.1.18.

Gómez-Trigueros I. M., Ruiz-Bañuls M. y Ortega-Sánchez, D. (2019). Digital Literacy of Teachers in Training: Moving from ICTs (Information and Communication Technologies) to LKTs (Learning and Knowledge Technologies). Education Sciences. 9(4): 274. DOI: https://doi.org/10.3390/educsci9040274.

Heredia Ponce, H., \& Romero Oliva, M. (2017). El blog como estrategia lectora en el aula de Secundaria. Revista de Estudios Socioeducativos. ReSed, (5), pp. 75-88. Recuperado de https://cutt.ly/PozmPua

Iglesias Martínez, M., Lozano Cabezas, I., \& Roldán Soler, I. (2018). La calidad e innovación educativa en la formación continua docente: un estudio cualitativo en dos centros educativos. Revista Iberoamericana de Educación, 77(1), 13-34. DOI: https://doi.org/10.35362/rie7713090.

Jeans, B. (2011). Canciones para Paula. Barcelona, Planeta. 
Jenkins, H. (2008). Convergence Culture: La cultura de la convergencia de los medios de comunicación. Barcelona: Paidós.

Kampylis, P., Devine, J., Punie, Y. \& Newman, T. (2016). Supporting schools to go digital: From a conceptual model towards the design of a self-assessment tool for digital-age learning. 9th annual International Conference of Education, Research and Innovation. The International Academy of Technology, Education and Development (IATED), 816-825. DOI: https://doi.org/10.21125/iceri.2016.1185.

Kress, G. y Van Leeuwen, T. (2001). Multimodal Discourse - The Modes and Media of Contemporary Communication. Londres: Arnold.

Kress, G., Jewitt, C., Ogborn, J. y Tsatsarelis, C. (2014). Multimodal teaching and learning. The Rhetorics of the Science Classroom. London-New York: Bloomsbury.

Larraz Rada, V., Álvarez Herrero, J. F., Espuny Vidal, C., González Martínez, J. (2019). La evaluación de la competencia digital y de la competencia digital docente. En Gisbert Cervera, M. Esteve-González, V., Lázaro Cantabrana, J. L. (Eds.) (2019). ¿Cómo abordar la educación del futuro? Conceptualización, desarrollo y evaluación de la competencia digital docente. Barcelona: Octaedro pp.169-182.

Lluch, G. (2019): Els booktubers en català. Un constructe virtual? Zeitschrift für Katalanistik, 32, 33-46 Recuperado de: https://cutt.ly/0ox3Jmo

Margallo, A. M. (2012). La educación literaria en los proyectos de trabajo. Revista Iberoamericana de Educación. Monográfico Didáctica de la Lengua y la Literatura, n.59, 139-156.

Meneses, J. Fàbregues, S., Jacovkis, J. y Rodríguez-Gómez, D. (2014). La introducción de las TIC en el sistema educativo español (2000-2010): un análisis comparado de las políticas autonómicas desde una perspectiva multinivel. Estudios sobre Educación,27, 63-90. DOI: https://doi.org/10.15581/004.27.63-90.

Mora, V.L. (2012). El lectoespectador. Barcelona: Seix Barral.

Pasqual, G. (2014). Xènia, tens un WhatsApp. Valencia: Barcanova.

Pérez-Torres, V., Pastor-Ruiz, Y. \& Abarrou-Ben-Boubaker, S. (2018). YouTuber videos and the construction of adolescent identity. [Los youtubers y la construcción de la identidad adolescente]. Comunicar, 55, 61-70. DOI: https://doi.org/10.3916/C552018-06.

Rekalde, I. y García, J. (2015). El aprendizaje basado en proyectos: un constante desafío. Innovación educativa, 25, pp. 219-234. DOI: http://doi.org/c6q9.

Ribera, J. y Martín, A. (2005). Todos los detectives se llaman Flanagan. Madrid, Anaya. 
Rovira-Collado, J. (2016). Mirar como maestros para el desarrollo de la comprensión lectora. Blogs educativos para la competencia profesional en futuros docentes, Investigaciones Sobre Lectura, 6, 58-75. DOI: https://doi.org/10.37132/isl.v0i6.154

Rovira-Collado, J. y Hernández Ortega, J. (2016). Aspectos de literacidad digital en el desarrollo de los Entornos Personales de Aprendizaje (PLE) en el área de Lengua y Literatura. En R. Roig-Vila (Coord.) Tecnología, innovación e investigación en los procesos de enseñanza-aprendizaje, Barcelona, Octaedro.

Rovira-Collado, J., Ambròs Pallarès, A. y Hernández Ortega, J. (2019). Innovación educativa y uso de las TAC en el Máster de Secundaria: propuestas formativas para docentes ecuatorianos en el programa UB-UNAE. Tejuelo, 30, 73-110. DOI: https://doi.org/10.17398/1988-8430.30.73.

Ruescas, J. (2013). Pulsaciones. Madrid, Ediciones SM.

Sánchez Rodríguez, S. y Cancelas-Ouviña, L. P. (2015). Tendencias educativas en DLL en el siglo XXI: ámbitos de investigación. En L. P. Cancelas-Ouviña y S. Sánchez Rodríguez (2015). Tendencias en educación lingüística. Granada: GEU, pp. 9-34.

Scolari, C. A. (2013). Narrativas transmedia. Cuando todos los medios cuentan. Barcelona: Deusto.

Scolari, C. A., Lugo, N. y Masanet, M. J. (2019). Educación Transmedia. De los contenidos generados por los usuarios a los contenidos generados por los estudiantes. Revista Latina de Comunicación Social, 74, 116-132. Recuperado de http://www.revistalatinacs.org/074paper/1324/07es.html. DOI: 10.4185/RLCS2019-1324.

Van Leeuwen, T. y Jewitt, C. (2001). The handbook of visual analysis. London: SAGE.

Zhang, L. y Cassany, D. (2019). The 'danmu' phenomenon and media participation: Intercultural understanding and language learning through The Ministry of Time. [El fenómeno «danmu» y la participación mediática: Comprensión intercultural y aprendizaje de lenguas a través de El Ministerio del Tiempo]. Comunicar, 58, 19-29. DOI: https://doi.org/10.3916/C58-2019-02. 